\title{
BMJ Open Health-related quality of life and anxiety in the PAN-CAN lung cancer screening cohort
}

\author{
Niloofar Taghizadeh, ${ }^{1}$ Alain Tremblay, ${ }^{1}$ Sonya Cressman, ${ }^{2}$ Stuart Peacock, ${ }^{2}$ \\ Annette M McWilliams, ${ }^{3}$ Paul MacEachern, ${ }^{1}$ Michael R Johnston, ${ }^{4}$ John Goffin, ${ }^{5}$ \\ Glen Goss, ${ }^{6}$ Garth Nicholas, ${ }^{6}$ Simon Martel, ${ }^{7}$ Francis Laberge, ${ }^{7}$ Rick Bhatia, ${ }^{8}$ \\ Geoffrey Liu, ${ }^{9}$ Heidi Schmidt, ${ }^{9}$ Sukhinder Atkar-Khattra, ${ }^{2}$ Ming-Sound Tsao, ${ }^{9}$ \\ Martin C Tammemagi, ${ }^{10}$ Stephen C Lam, ${ }^{2}$ for the Pan-Canadian Early Lung Cancer \\ Study Group
}

To cite: Taghizadeh $\mathrm{N}$, Tremblay A, Cressman S, et al. Health-related quality of life and anxiety in the PAN-CAN lung cancer screening cohort. BMJ Open 2019;9:e024719. doi:10.1136/ bmjopen-2018-024719

- Prepublication history and additional material for this paper are available online. To view these files, please visit the journal online (http://dx.doi. org/10.1136/bmjopen-2018024719).

Received 28 June 2018 Revised 19 0ctober 2018 Accepted 6 December 2018

Check for updates

(C) Author(s) (or their employer(s)) 2019. Re-use permitted under CC BY-NC. No commercial re-use. See rights and permissions. Published by BMJ.

For numbered affiliations see end of article.

Correspondence to

Dr Alain Tremblay;

atrembla@ucalgary.ca

\section{ABSTRACT}

Objectives The impact of lung cancer screening with low-dose chest CT (LDCT) on participants' anxiety levels and health-related quality of life (HRQoL) is an important consideration in the implementation of such programmes. We aimed to describe changes in anxiety and $H R Q 0 L$ in a high-risk Canadian cohort undergoing LDCT lung cancer screening.

Methods 2537 subjects who had $2 \%$ or greater lung cancer risk over 6 years using a risk prediction tool were recruited from eight centres across Canada in the Pan-Canadian Early Detection of Lung Cancer Study (2008-2010). We compared HRQoL and anxiety levels before and after screening of 1237 participants with LDCT (excluding a subset of 1300 participants who also underwent autofluorescence bronchoscopy screening), as well as after investigations performed because of a positive screening examination. The 12-item short-form Physical and Mental Component Scales (SF-12), EQ-5D-3L scores and State Trait Anxiety Inventory-State anxiety were used at each assessment.

Results Overall, there were no clinically significant differences in HRQoL outcomes between baseline and each of the survey time points following initial screening. No mean change in anxiety in the overall cohort was noted following baseline LDCT, but more participants had clinically significant increase in anxiety versus decrease after baseline screening (increase $>$ minimal clinically important difference (MCID) $(n=180)$ vs decrease $>$ MCID $(n=50), p<0.001)$. This finding persisted but to a lesser degree at the 12 month time point (increase $>$ MCID $(n=146)$ vs decrease $>\operatorname{MCID}(n=87), p<0.001)$. Conclusions CT screening for lung cancer has no major overall impact on HRQoL among participants, although a minority of participants (number-needed-to-harm $=7$ after baseline screening and 18 at 1 year) demonstrated clinically significant increased anxiety levels. Trialregistration number NCT00751660; Results.

\section{INTRODUCTION}

Lung cancer is the leading cause of cancer death in North America and around the

\section{Strengths and limitations of this study}

- This study is the first to describe the psychological and quality of life impacts of lung cancer screening on discrete individuals undergoing low-dose CT examinations.

- This allows the calculation of number-needed-toharm estimates based on the minimal clinically significant difference of each instrument rather than mean group changes, important in the informed decision-making process with individuals considering this intervention.

- Our cohort was drawn from a multicentre study with high follow-up rates using a participant's baseline status to detect any changes post-screening.

- Limitations include the lack of an unscreened control group and the relative homogeneity of our participants (Canadian, Caucasian).

world. ${ }^{1}$ Early detection and treatment of lung cancer through screening is a promising strategy to reduce lung cancer mortality. ${ }^{2}$ The largest trial performed to date, the National Lung Screening Trial (NLST), demonstrated that low-dose CT (LDCT) screening in highrisk individuals (ie, ever smokers aged 55-74 years, $\geq 30$ pack-years (number of cigarettes per day/20×number of years of smoking) and $<15$ years since quitting) of smoking significantly reduced lung cancer and overall mortality. ${ }^{3}$ American and Canadian preventative healthcare agencies have since published recommendations in favour of LDCT lung cancer screening. ${ }^{3}{ }^{4}$ However, no screening intervention is without potential harm, including adverse psychological impact of the screening intervention, screening results, or subsequent investigations in most participants who will not be found to have cancer. Potential detriments of lung cancer screening 
include anxiety and distress from the evaluation of both CT detected false positive and overdiagnosed cancers. A small proportion of the screen-detected tumours would never lead to clinical symptoms, but these overdiagnosed lung cancers are frequently treated, with associated risks of adverse effects. ${ }^{56}$ Moreover, studies have shown that CT lung screening has a high rate of significant lung cancer-unrelated incidental findings (SIFs). ${ }^{7}$ These SIFs may require additional investigations and therefore can be associated with adverse psychological impact on participants in a screening programme. ${ }^{6}$

A recent systematic review on the psychological burden of LDCT revealed that LDCT screening may be associated with a short-term psychological burden in participants. $^{8}$ Studies to date have explored mean changes in groups of individuals rather than rates of clinically significant changes in individuals screened. Effective policy decisions regarding the implementation of lung cancer screening and informed decision making by individuals requires reliable evidence on its potential impacts on health-related quality of life (HRQoL) and psychological well-being of individual participants. ${ }^{9}$ Therefore, this study aimed to evaluate the impact of screening modalities on the quality of life and anxiety of participants in the Pan-Canadian Early Detection of Lung Cancer Study.

\section{METHODS}

\section{Study design and population}

The Pan-Canadian Early Detection of Lung Cancer Study, which has been described in detail previously, ${ }^{10} 11$ enrolled current or former smokers aged between 50 and 75 years and with a $2 \%$ or greater lung cancer risk over 6 years using a risk-prediction model developed using Prostate,
Lung, Colorectal and Ovarian Cancer Screening Trial data. ${ }^{12}$ Participants were recruited in eight centres across Canada (Calgary, Halifax, Hamilton, Laval, Ottawa, St-John's, Toronto and Vancouver) from September 2008 to December 2010 with each centres' institutional review board approving the study (online supplementary e-Appendix 1). Signed informed consent was obtained from each participant.

All participants were offered baseline LDCT with repeat screening at year 1 and 4 in addition to LDCT scans as appropriate for nodule follow-up, with the first half of the recruited subjects to receive autofluorescence bronchoscopy (AFB) as an additional screening modality. ${ }^{13}$ However, since AFB does not appear effective in the screening environment, ${ }^{13}$ and to avoid the potential confounding impact of AFB on HRQL, participants in the AFB arm of the study are excluded from the current analysis.

LDCT scan follow-up protocol were determined by the maximum long-axis diameter of the largest nodule identified. Participants with any semisolid or solid nodule $5-10 \mathrm{~mm}$ or ground-glass opacity $8-10 \mathrm{~mm}$ were to receive an additional LDCT at 3 months, with larger lesion being referred for clinical consultation. Any participant requiring repeat LDCT or investigation for a lung lesion other than a planned 12-month follow-up examination were considered to have a positive screening exam for the purpose of this analysis (figure 1). Participants were informed of the various possible findings which may be found on CT examinations and general protocols for investigations at the time of study consent. Individualised results letters with description of findings appropriate for a non-medical reader were developed by each study site.

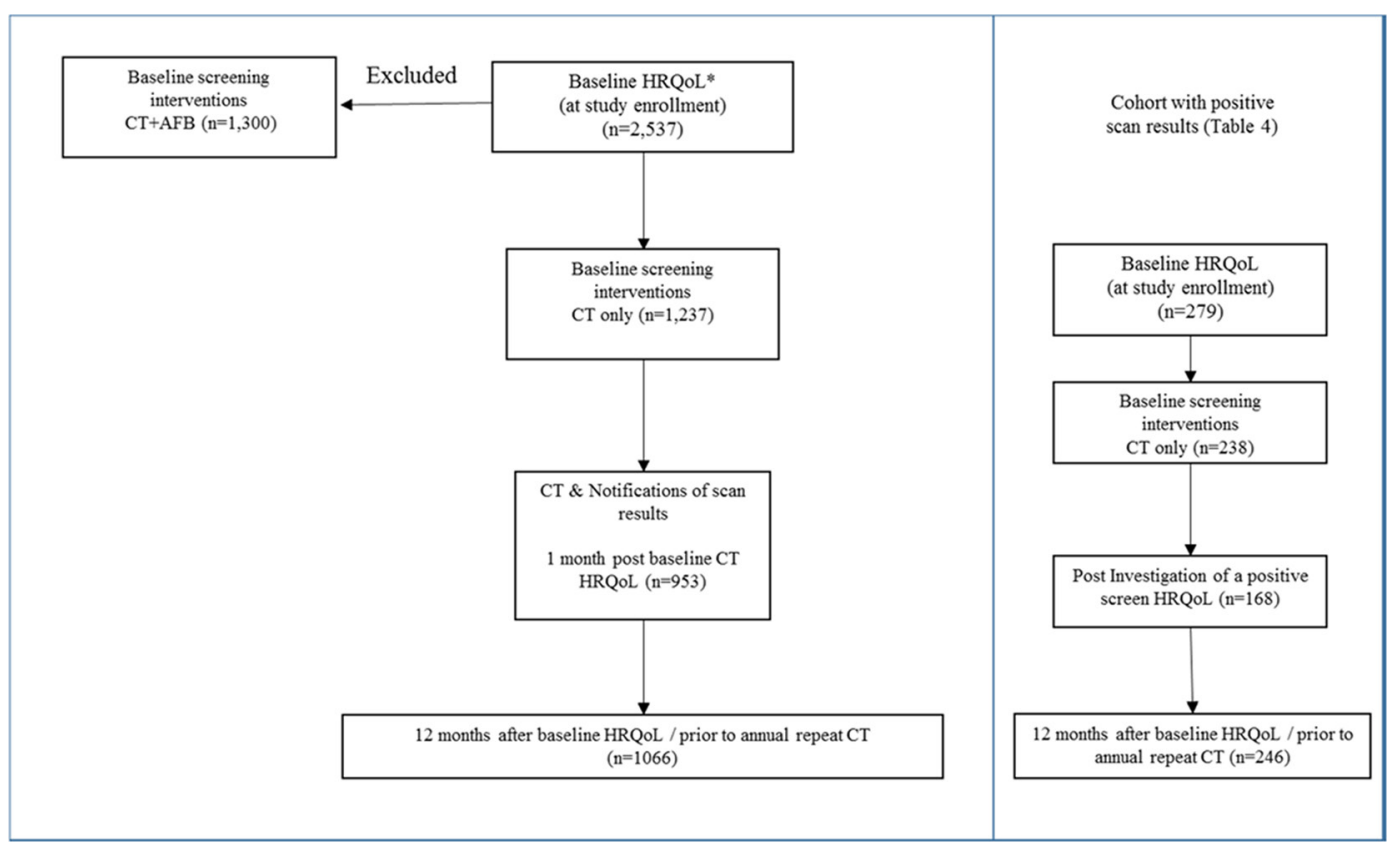

Figure 1 Assessments of the health-related quality of life (HRQoL) and anxiety in the Pan-Canadian Early Detection of Lung Cancer Study. 


\section{HRQoL and anxiety}

The 12-item short-form (SF-12) Physical Component Scales (PCS) and Mental Component Scales (MCS) ${ }^{14}$ and the EuroQoL questionnaire (EQ-5D-3L (three-level version of EQ-5D)) were used to determine the participants' HRQoL at each assessment. The test-retest reliability coefficient is reported to be 0.89 for the PCS and 0.76 for the MCS. The EQ-5D-3L consists of a preference-based index score and a Visual Analogue Scale (VAS); the index scores were derived from the current Canadian $\operatorname{tariff}^{15}$ (a maximum (best) value of 1 (for health state 11111) and a minimum value of -0.34 (for 33333)). The VAS is a Likert scale asking participants to draw a line to their current health status on a visual scale ranging between 0 and 100. Scores on the SF-12 are standardised (ie, mean $=50$ and $\mathrm{SD}=10$ ), with a higher score indicating better HRQoL.

To evaluate potential anxiety induced by the results of the screening tests, we used the Spielberger State Trait Anxiety Inventory Form Y (STAI) ${ }^{16}$ Additional methodology details are provided in the online supplementary file.

The questionnaires were administered in person at the time of study enrolment (baseline), then by phone within 1 month after the CT results were received by the participants, 1 month after any additional follow-up CT scan or other testing following a positive screen (postinvestigations) and prior to the first annual repeat LDCT (12 months post-baseline) (figure 1).

\section{Statistical analyses}

Descriptive analyses of the participants' characteristics and screening outcome were performed. We calculated summary scores of outcome measures for participants in each category at each of the study time points (at baseline, 1 month post-baseline CT scan, 12 months after baseline and postinvestigations). In addition, the above scores were compared separately in the subset of participants with a positive screening intervention.

To compare overall differences in HRQoL and state-anxiety scores between baseline and each of the survey time points, generalised linear mixed models were used to take into account the clustering of data within the eight study sites, the repeated measurement of each individual, the non-normally distributed/skewed outcomes and any missing data. The estimated margin of means with adjustment for multiple comparisons (Bonferroni correction) was calculated to contrast baseline versus each of the study time points. In these estimations, margins involving empty cells were treated as not estimable. When significant long-term differences were noted in our mixed model, we further explored the factor association with the observed changes using a multivariate regression model with adjustment for scan results, age, gender, self-reported race, smoking status, pack-years, alcohol consumption, education, family history of any cancer, participants' concern about getting lung cancer at baseline and for the clustering of data within eight study sites.
We further compared the proportion of individuals with improvement versus deterioration greater than the minimal clinically important difference (MCID) for each instrument. MCIDs for outcome measures were selected based on previously published results as follows: $\mathrm{EQ} \mathrm{VAS}=8,{ }^{17} \mathrm{EQ}-5 \mathrm{D}-3 \mathrm{~L}$ index values $=0.05,{ }^{18} \mathrm{PCS}=8.1,{ }^{19}$ MCS $=4.7,{ }^{14}$ STAI-State Anxiety $=10 .{ }^{20}$ The comparisons between these two proportions were performed using Z-test and if significant, the excess number of cases with improvement versus worsening scores were calculated as a percentage of cases with available data. When significant differences were noted, a number-needed-to-harm $(\mathrm{NNH})$ or number-needed-to-treat (NNT) calculation was applied as appropriate (total number of case/excess cases with worsened or improved score).

Two-sided $\mathrm{p}$ values $<0.05$ were considered as statistically significant. All analyses were performed using SPSS V.24 or STATA V.14. Sample size was determined by other primary study factors relating to the screening intervention and not the current analysis.

\section{Patient and public involvement}

Patient and public involvement in the design of the research was included through the main funding agencies collaborating on the project. This includes the Terry Fox Research Institute, the research arm of The Terry Fox Foundation. In addition, public input was obtained through involvement of the Canadian Partnership Against Cancer, an independent organisation funded by the federal government to accelerate action on cancer control for all Canadians. Patients were not specifically involved in the recruitment and conduct of the study and no specific plan to disseminate research findings to participants has been made.

\section{RESULTS}

\section{Participant characteristics}

Two thousand five hundred and thirty-seven participants were enrolled in the Pan-Can study, and 1237 underwent LDCT alone (without AFB). The mean (SD) age of these participants was $62.9(6.1)$ at baseline. Males $558(45.1 \%)$, Caucasian $1201(97.1 \%)$, current smokers $768(62.1 \%)$ and regular alcohol drinkers $961(77.7 \%)$ comprised the largest groups of participants. The median (IQR) packyears of smokers was 51.3 (21.6) and mean (SD) duration of smoking was 43.9 (6.1) years. A family history of lung cancer was present in 392 participants (31.7\%) (table 1). Median (IQR) lung cancer risk score was 3.5\% (2.9) over 6 years. Positive baseline LDCT examinations were noted in $279(22.6 \%)$ participants of which $35(2.8 \%)$ led to a diagnosis of lung cancer.

\section{Health-related quality of life and anxiety measures Baseline}

At baseline, participants reported being concerned about getting lung cancer always $(6.1 \%)$, often $(19.0 \%)$ and sometimes $(53.0 \%)$. General health problems were 
Table 1 Baseline characteristics of Pan-Canadian Early Detection of Lung Cancer Study participants

\begin{tabular}{lc}
\hline Characteristics & All enrolled (n=1237) \\
\hline Age, mean (SD) & $62.9(6.1)$ \\
\hline Gender (males), $\mathrm{n}(\%)$ & $558(45.1)$ \\
\hline Race ${ }^{\star}, \mathrm{n}(\%)$ & $1201(97.1)$ \\
\hline Caucasian & $15(1.2)$ \\
\hline Asian & $7(0.6)$ \\
\hline Black or African Canadian & $4(0.3)$ \\
\hline Aboriginal & $0(0.0)$ \\
\hline Pacific Islander & $10(0.8)$ \\
\hline Other & \\
\hline Education, $\mathrm{n}$ (\%) & $32(2.3)$ \\
\hline Eighth grade or less & $153(12.4)$ \\
\hline Ninth to 12th grade & $337(27.2)$ \\
\hline High school graduate & $107(8.7)$ \\
\hline Bachelor's degree & $260(21.0)$ \\
\hline Technical/vocational/school & \\
certificate & $205(16.6)$ \\
\hline Associate degree/some college & $144(11.6)$ \\
\hline Advanced Degree & \\
\hline Smoking habits & $768(62.1)$ \\
\hline Current smokers, $\mathrm{n}$ (\%) & $51.3(21.6,2.2-230)$ \\
\hline Pack-years, median (IQR, range) & $43.9(6.1)$ \\
\hline $\begin{array}{l}\text { Smoking duration (years), mean } \\
\text { (SD) }\end{array}$ & \\
Alcol & \\
\hline
\end{tabular}

Alcohol consumption

Current regular drinkerst, $\mathrm{n}(\%) \quad 961(77.7)$

Family history of lung cancer, $\mathrm{n}(\%) \quad 392(31.7)$

Being worried about getting lung cancer, $\mathrm{n}(\%)$

\begin{tabular}{lc} 
Rarely or never & $267(21.6)$ \\
\hline Sometimes & $656(53.0)$ \\
Often & $235(19.0)$ \\
All of the time & $75(6.1)$ \\
Scan results at baseline, $\mathrm{n}(\%)$ & \\
$\quad$ Positive & $279(22.6)$ \\
$\quad$ Negative & $958(77.4)$ \\
$\begin{array}{l}\text { Lung cancer risk score, median } \\
\text { (IQR, range) }\end{array}$ & $3.5(2.9,2.0-33.5)$ \\
\hline
\end{tabular}

${ }^{*}$ Missing, $n(\%)=5(0 \cdot 2)$.

†Regular alcohol consumption: having more than one drink per week for a period of 6 months or more. Missing, $n=11$.

reported by $65.0 \%$ of respondents on at least one item on the EQ-5D-3L. Average baseline EQ VAS, EQ-5D-3L index values, PCS, MCS and STAI-State Anxiety scores were $76.3,0.84,46.1,51.1$ and 30.9 , respectively (table 2 ).

\section{Baseline screening}

No statistically significant mean changes in EQ VAS, EQ-5D-3L index values, PCS or MCS levels were noted following baseline CT screening. In addition, the proportion of individuals experiencing a deterioration versus improvement greater than the MCID for $\mathrm{EQ}$ VAS (figure 2), EQ-5D-3L (figure 3), PCS (figure 4) and MCS (figure 5) were not significantly different. However, the STAI-State Anxiety levels increased in participants following baseline LDCT (change (95\% CI): 2.27 (0.57 to 3.96 ), $p$ value $<0.001$ ) (table 2 ). A greater proportion of individuals experiencing a deterioration versus improvement greater than the MCID of 10 for the STAI-State Anxiety levels was also noted (increase >MCID $(n=180)$ vs decrease $>$ MCID $(n=50), p$ value $<0.001)$ (figure 6). The excess number of participants with increased versus decreased anxiety represents $13.8 \%((180-50) / 937$, $\mathrm{NNH}=7)$ of participants with available data. This change remained significant even if only participant with a negative screen were considered (mean baseline STAI 31.2; increase $>$ MCID $(n=129)$ vs decrease $>$ MCID $(n=40)$, $p$ value $<0.0001)$. Multivariate regression analysis demonstrated female gender and increased baseline concern about getting lung cancer to be associated with increased anxiety following screening (table 3 ).

\section{Twelve-month assessment}

No statistically significant mean changes in EQ VAS, EQ-5D-3L index values, PCS or STAI-State Anxiety levels were detected in participants at the 12-month interview. The proportion of individuals with deterioration versus improvement greater than the MCID for the instrument remained significant for the STAI-State anxiety levels (increase $>$ MCID $(n=146)$ vs decrease $>$ MCID $(n=87)$, $\mathrm{p}$ value $<0.0001)$, representing $5.5 \% \quad((146-87) / 1066$, $\mathrm{NNH}=18$ ) of participants (figure 6). The proportion of individuals experiencing a deterioration versus improvement greater than the MCID for EQ VAS (figure 2), EQ-5D-3L (figure 3), PCS (figure 4) and MCS (figure 5) were not significantly different.

\section{Positive screen and investigation}

Among participants receiving a positive scan results $(n=279)$, no statistically significant mean changes in EQ VAS, EQ-5D-3L index values, PCS or MCS were detected following baseline LDCT (online supplementary table 1). However, more participants experienced a clinically significant decrease versus increase in anxiety score (increase $>$ MCID $(\mathrm{n}=20)$ vs decrease $>$ MCID $(\mathrm{n}=41), \quad \mathrm{p}$ value $=0.002) \quad$ representing $8.8 \% \quad((41-$ 20) $/ 238, \mathrm{NNT}=11$ ) of these participants (figure 7 ). This decreased anxiety persisted at the 12-month interview (increase $>$ MCID $(n=14)$ vs decrease $>$ MCID $(n=35)$, $\mathrm{p}$ value $=0.003) \quad$ representing $8.5 \% \quad((35-14) / 246$, $\mathrm{NNT}=12$ ) of participants (figure 8 ).

Following investigation examinations, no statistically significant mean changes in EQ VAS, EQ-5D-3L index values, PCS, MCS or anxiety were detected. Postinvestigation changes revealed no statistically significant changes in the proportion of individuals with deterioration versus improvement greater than the MCID (figure 7). 
Table $2 \mathrm{HRQOL}$ and anxiety measures at baseline and at different time points within the study (generalised linear mixed model)

\begin{tabular}{|c|c|c|c|}
\hline & Baseline $(n=1237)$ & $\begin{array}{l}1 \text { month post-baseline CT } \\
\text { scan mean, change }(95 \% \mathrm{Cl})(\mathrm{n}=953)\end{array}$ & $\begin{array}{l}12 \text { months after baseline CT mean, } \\
\text { change }(95 \% \mathrm{Cl})(\mathrm{n}=1066)\end{array}$ \\
\hline EQ VAS* & 76.3 & $76.8,0.42(-1.39$ to 2.23$)$ & $76.8,0.22(-0.88$ to 1.32$)$ \\
\hline SF-12: PCS† & 46.1 & $46.8,0.61(-0.15$ to 1.37$)$ & $46.4,0.31(-0.55$ to 1.17$)$ \\
\hline SF-12: MCS & 51.1 & $50.9,-0.26(-1.04$ to 0.52$)$ & $51.2,-0.14(-1.14$ to 0.86$)$ \\
\hline
\end{tabular}

`EQ VAS: 'We would like to know how good or bad your health is today' (100-best imaginable, 0-worst imaginable). †Physical Health Composite Scores (US population mean $=50 \pm 10$ ), with higher score corresponding to better state. ¥Mental Health Composite Scores (US population mean $=50 \pm 10$ ), with higher score corresponding to better state. §STAI-State score $>39$ considered clinically significant symptoms.

ПP value $<0.05$ compared with baseline. Postestimated marginal means with adjustment for multiple comparison (Bonferroni). EQ-5D-3L, EuroQol-5D three-level version; HRQoL, health-realted quality of life; PCS, physical component scale; MCS, mental component scale; SF-12, 12-item short-form; STAI, State Trait Anxiety Inventory; VAS, visual analogue scale.

The proportion of different levels of each questionnaires' dimensions by study visits, as well as number of missing values, are shown in the online supplementary tables 2-4.

\section{DISCUSSION}

This study offers detailed information on HRQoL and anxiety following LDCT for lung cancer screening in a Canadian high-risk selected population using validated assessment tools measuring overall HRQoL as well as specific physical, psychological and anxiety scores. Our study found no clinically significant differences in HRQoL outcomes between baseline and each of the survey time points following initial screening in the cohort as a whole. However, more participants experienced a clinically significant increased anxiety (vs decreased) following baseline LDCT. This finding was more pronounced among females and participants who were concerned about getting lung cancer at baseline. Paradoxically, decreased anxiety was more frequent in the subgroup with positive baseline scan, although the impact of scan results did not reach statistical significance in the multivariate analysis. Over the long term, no adverse effects on HRQoL were noted but some of the excess in increased anxiety levels persisted.

In line with our findings, analyses of other screening cohorts including the National Lung Screening Trial (NLST) ${ }^{21}$ Dutch-Belgian Lung Cancer Screening Trial (NELSON), ${ }^{22}$ Prostate, Lung, Colorectal, and Ovarian Cancer Screening Trial (PLCO) ${ }^{23}$ and United Kingdom Lung Cancer Screening Trial(UKLS), ${ }^{24}$ as well as two recent meta-analyses have demonstrated that lung cancer screening is associated with little to no adverse physical or psychological long-term impact on participants. ${ }^{825}$ While analysis of the Danish Lung Cancer Screening Trial did show negative consequences at 1 year ${ }^{26}$ and 2 year $^{27}$ follow-up, the degree of change was actually greater in the control (no screening) arm of the trial.

Our finding of decreased anxiety following a positive screen is in contrast with those reported in the UKLS ${ }^{24}$ and NLST trials, ${ }^{21}$ which observed a short-term increase

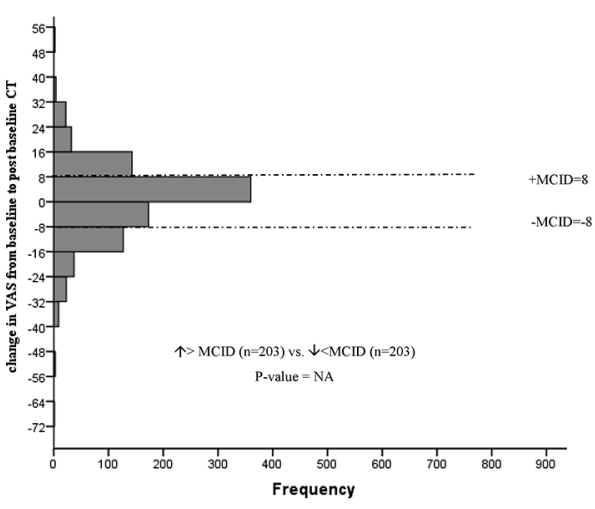

(A)

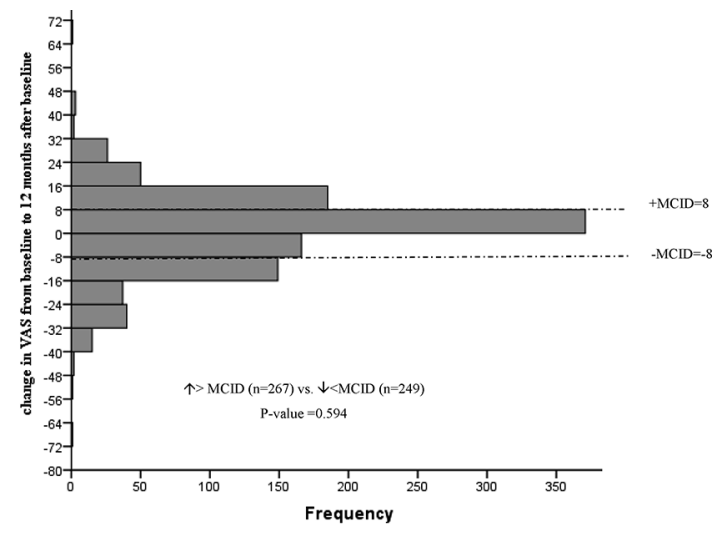

(B)

Figure 2 Changes in EuroQoL VAS from baseline to post-baseline CT (A), and 12 months after baseline (B). MCID, minimal clinically important difference; VAS, visual analogue scale. 


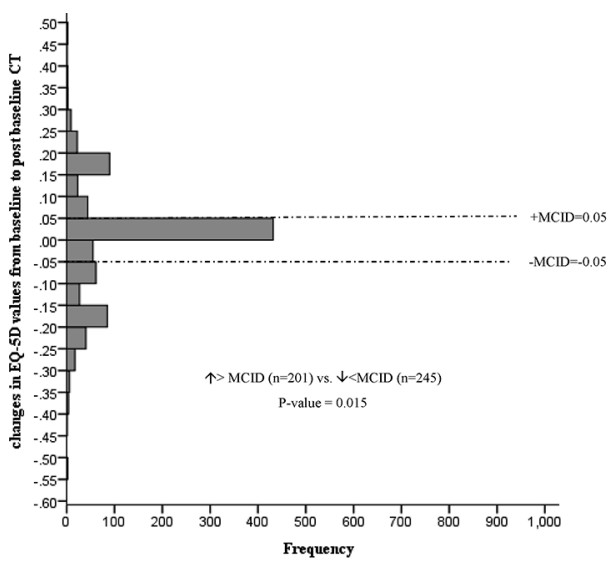

(A)

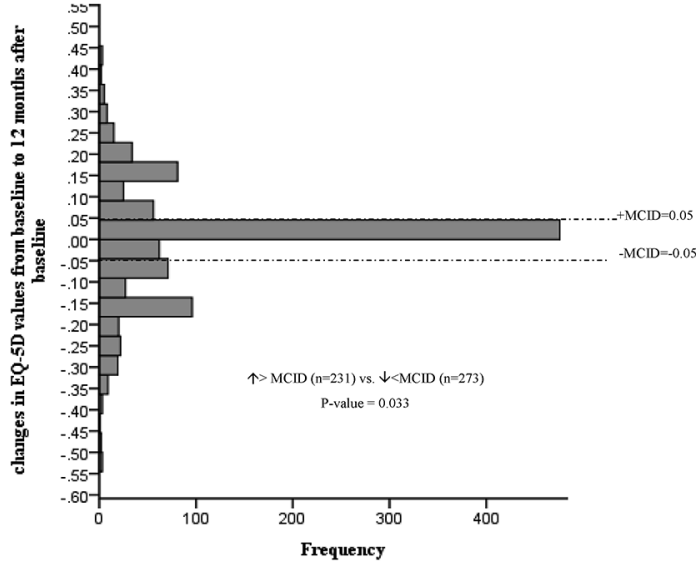

(B)

Figure 3 Changes in EuroQoL(EQ)-5D-3L from baseline to post-baseline $C T(A)$, and 12 months after baseline (B). EQ-5D-3L, EuroQoL 5D-three-level version; MCS, mental component scale; MCID, minimal clinically important difference.

in distress levels 2 weeks or 2 months, respectively, after a positive result notification of baseline screening. Results from NELSON ${ }^{28}$ and from the Pittsburgh Lung Screening Study $^{29}$ also reported a short-term lung cancer-specific distress, a poorer quality of life and a higher level of anxiety among participants with indeterminate scan results compared with those with negative results. However, in both studies, these negative impacts disappeared over time. The explanation for this finding may relate to the small size of our programme and personalised communication process for results in the study. The absolute number of participants with significant changes in this metric was also relatively small, so that this finding should not be overinterpreted.

Similar to our findings, NELSON study reported a worse HRQoL outcomes among females compared with males. ${ }^{28}$ Furthermore, our observation regarding females is also consistent with the results of a study of PLCO participants $^{23}$ that found a poorer MCS outcomes in females compared with males.

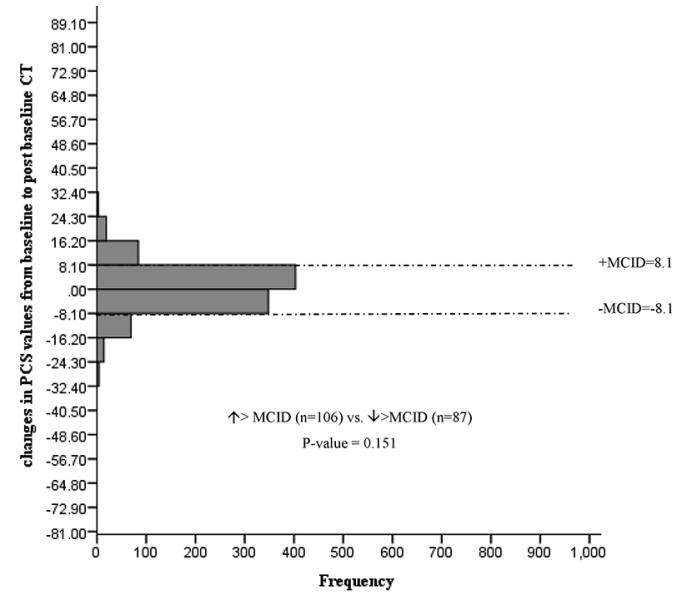

(A)
In most studies reported to date, statistically significant mean changes in HRQoL-related scores detected in groups of screened individuals have been small and of questionable clinical significance limiting the impact of such findings in clinical decision-making. Conversely, lack of statistically significant changes in population means can mask clinically meaningful changes in individuals. The MCID has been suggested to be a useful benchmark to define the smallest difference in HRQoL that individuals perceive as beneficial or harmful and that mandates a change in management. ${ }^{30}$ Only two previous lung cancer screening trial have reported MCID levels to interpret the changes in HRQoL of participants. ${ }^{22} 2428$ However, both applied this concept to mean population changes rather than to discrete individual changes. Our study is unique in providing discrete participant data on the proportion of individuals with improvement versus deterioration greater than the MCID for each assessment tool. This has allowed us to attribute to the intervention excess cases of deterioration versus improvement given normal expected

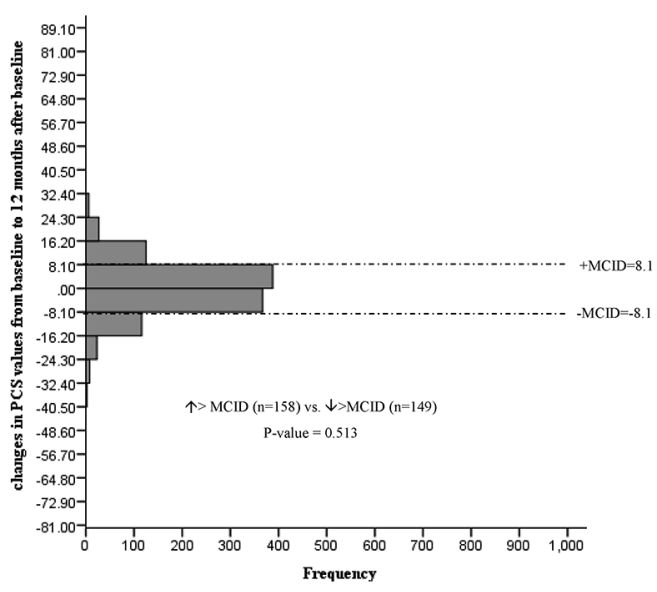

(B)

Figure 4 Changes in 12-item short-form PCS from baseline to post-baseline CT (A) and 12 months after baseline (B). PCS, Physical Component Scale; MCID, minimal clinically important difference; 


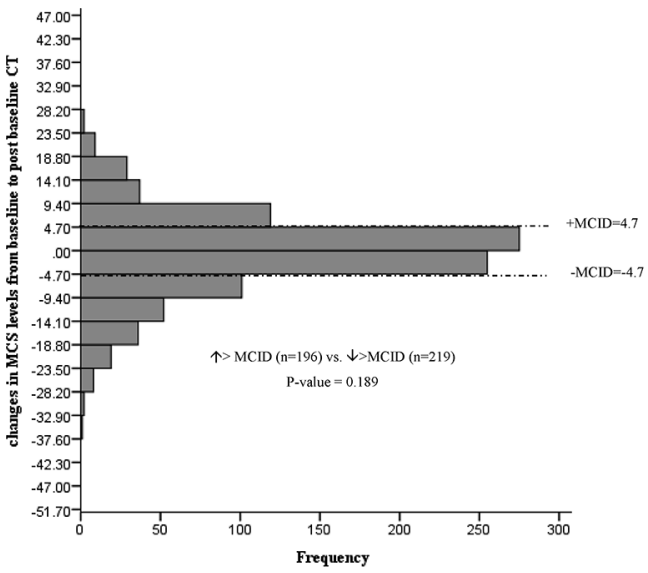

(A)

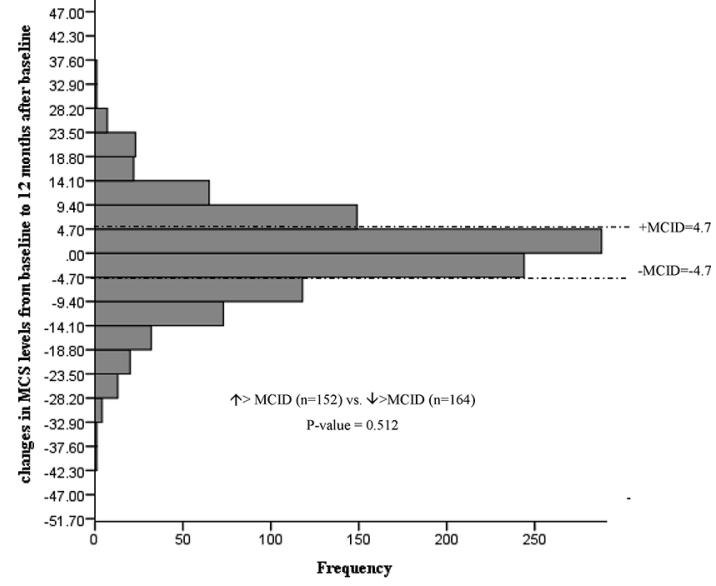

(B)

Figure 5 Changes in 12-item short-form MCS from baseline to post baseline CT (A) and 12 months after baseline (B). MCS, Mental Component Scale; MCID, minimal clinically important difference;

variations in each individual over time. This can suggest if a true clinically significant impact is present, and specifying how many individuals are impacted by such a change, in order to calculate a 'NNH' value. With this approach, we found that the proportion of individuals with improvement versus deterioration greater than the MCID for the STAI was significantly different among all participants with a NNH of 7 in the short term following screening, and 18 at 1 year post-screening. Our data adds to an evolving body of evidence which suggests that LDCT screening for lung cancer does not have overall significant negative impacts on the HRQoL of the population screened. However, a minority of individuals do experience small but clinically significant increases in anxiety levels following screening.

The major strengths of our study include the use of a large multicenter sample of eligible participants and reporting of individual participant data in relation to their MCID using three different and well-established instruments for measuring HRQoL and anxiety as well

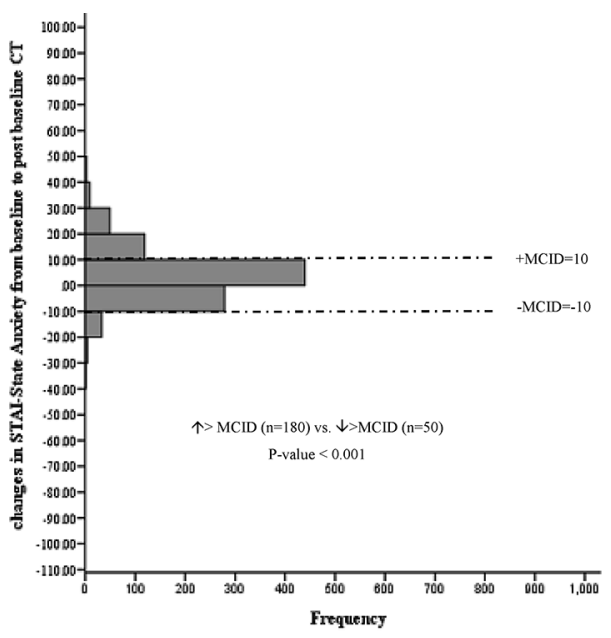

(A) as the risk prediction model used for the recruitment. ${ }^{31}$ Another strength of our study is the longitudinal design with a high follow-up and response rate (see online supplementary tables 2-4), which enabled us to assess short-term and long-term outcomes at different time points during screening process with each participant serving as his/her own control. While we enrolled a highrisk cohort using a risk prediction model, our participants' baseline HRQoL metrics appeared comparable to those of similarly aged individuals in the general population (adults aged 55-69, mean EQ VAS: $76^{32}$; age 50-59, mean STAI: 32.2 (female) $/ 34.5$ (male) ${ }^{16}$; age 50-69, PCS: $50.9-51.3$, MCS: $\left.50.7-50.9^{33}\right)$ suggesting that our findings could be generalisable to a broader population of screen-eligible individuals but with lower risk of lung cancer than in our population. Our study is also the first to use the full EQ-5D score in this population, which can be used to calculate quality-adjusted-life-years.

The current study has potential limitations. Our population was made up almost entirely of Caucasians, so that a

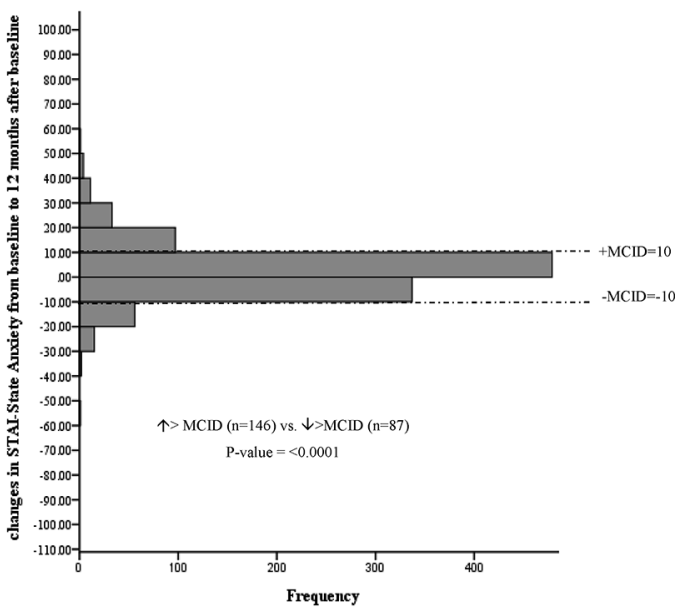

(B)

Figure 6 Changes in Spielberger STAI from baseline to post-baseline CT (A), and 12 months after baseline (B). MCID, minimal clinically important difference; STAI, State Trait Anxiety Inventory. 
Table 3 Factor associated with changes in anxiety levels from baseline to 1 month post-baseline CT scan

Changes in anxiety levels (STAI-S), Beta coefficient (95\% Cl)

\begin{tabular}{|c|c|}
\hline Positive scan results & $-0.70(-1.91$ to 0.51$)$ \\
\hline Age & $-0.09(-0.18$ to 0.01$)$ \\
\hline Females & $1.01(0.02 \text { to } 2.16)^{*}$ \\
\hline Current smokers & $0.57(-0.50$ to 1.64$)$ \\
\hline Pack-years & $-0.01(-0.03$ to 0.01$)$ \\
\hline Current alcohol consumption & $-0.63(-1.86$ to 0.60$)$ \\
\hline Family history of any cancer & $-1.14(-2.28$ to 0.01$)$ \\
\hline \multicolumn{2}{|c|}{ Participants' concern about getting lung cancer } \\
\hline All the time & $3.79(0.24 \text { to } 7.32)^{*}$ \\
\hline Often & $1.73(-0.00$ to 3.47$)$ \\
\hline Sometimes & $0.99(-0.12$ to 2.10$)$ \\
\hline
\end{tabular}

Multivariate regression model with adjustment for scan results, age, gender, race, smoking status, pack-years, alcohol consumption, education, family history of any cancer, participants' concern about getting lung cancer at baseline, and for the clustering of data within eight study sites. ${ }^{\star} \mathrm{P}<0.05$.

differential impact of screening on other ethnic communities cannot be determined. Owing to the study design for HRQoL assessments, we were unable to address the impacts of incidental findings on HRQoL and anxiety of participants. Another potential limitation is that we did not compare our results to an unscreened control group but instead used each participant's baseline scores. As such, other factors unrelated to the screening intervention, such as ageing or changes in smoking status, could affect the longitudinal changes (or lack thereof) noted in our study. ${ }^{34}$ However, two previous studies with a randomised design and a control group reported the HRQoL results that were comparable to our findings. ${ }^{14}{ }^{20}$ Another potential limitation is that the EQ-5D-3L is usually associated with a ceiling effect (ie, scores recording perfect health) ${ }^{35}$ and has limited ability to determine small changes in health status compared with the five-level EQ-5D-5L, which might offer improved measure of population-weighted health state utility. ${ }^{36}$ In our study, $35 \%$ of participants reported perfect scores on EQ-5D-3L at baseline, suggesting a ceiling effect that was adjusted for with a generalised linear mixed modelling approach. ${ }^{38}$ Moreover, HRQoL in our study was also measured by SF-12, which has been known to demonstrate a smaller ceiling/floor effect compared with EQ-5D-3L. ${ }^{35}$ In our study, no ceiling/floor effect was observed for the SF-12 scores. Finally, the statistical power to detect changes in some participant subgroups such as those with positive screens may be limited because of low number of participants with a positive scan results. Therefore, caution should be used in drawing conclusions.

The complexity of longitudinal analysis of HRQoL and the lack of agree on standardised approach compromise

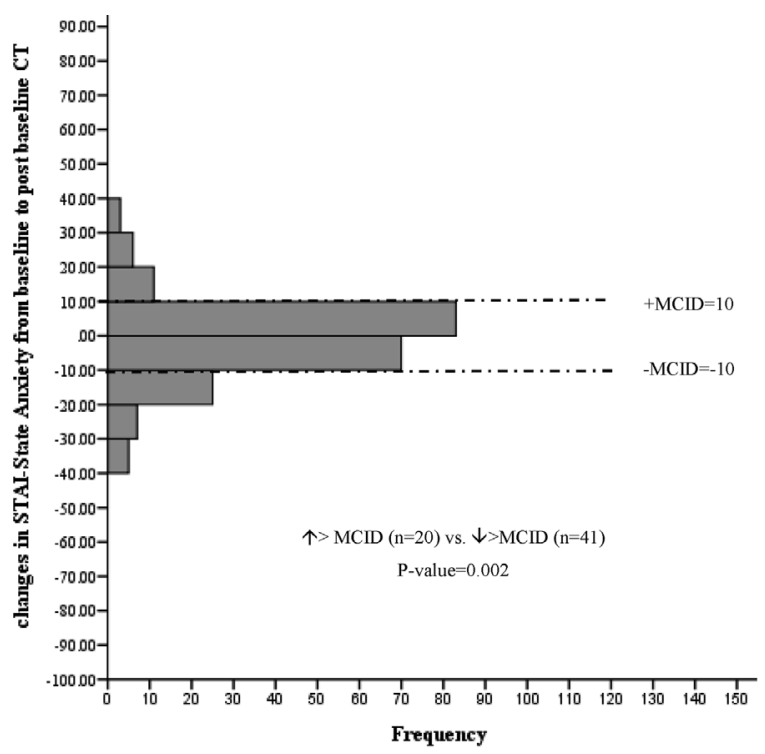

(A)

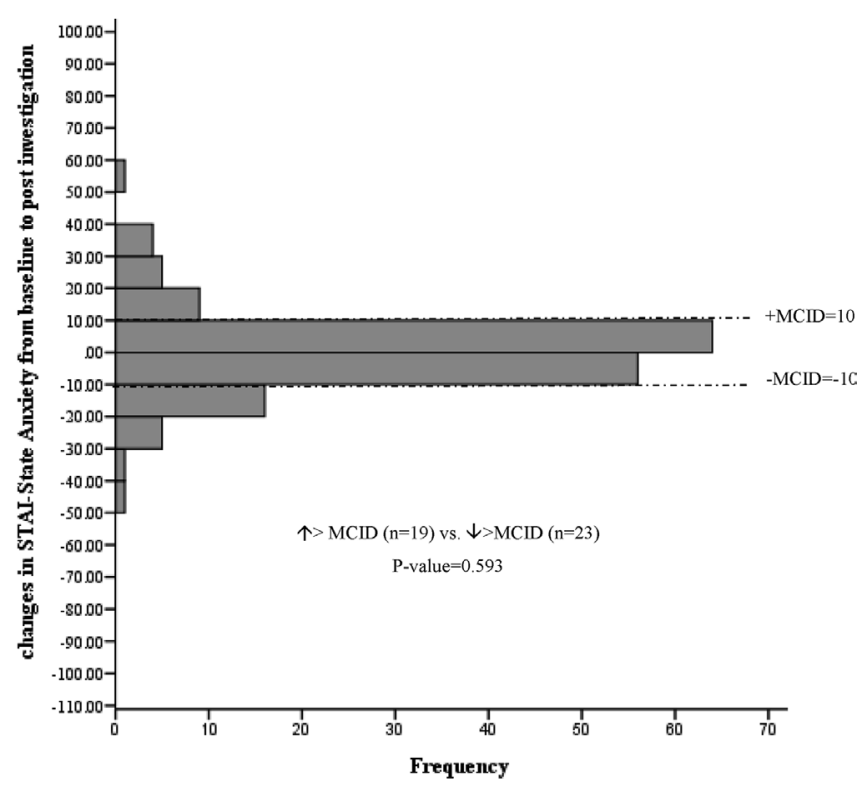

(B)

Figure 7 Changes in Spielberger STAl from baseline to post-baseline CT (A) and postinvestigation (B) among participants with a positive scan results. MCID, minimal clinically important difference; STAI, State Trait Anxiety Inventory.

the comparison of results between studies. ${ }^{39}$ Even the specific MCID level for each instrument can be debated. Ideally, such levels are determined in the specific population of interest, but such information is rarely available. Levels chosen for our analysis were determined prior to any data analysis based on best evidence for each instrument. As a confirmatory step, MCIDs selected in this study were found to approximate estimates obtained as half a SD (MID) of HRQoL measures in our population (results 


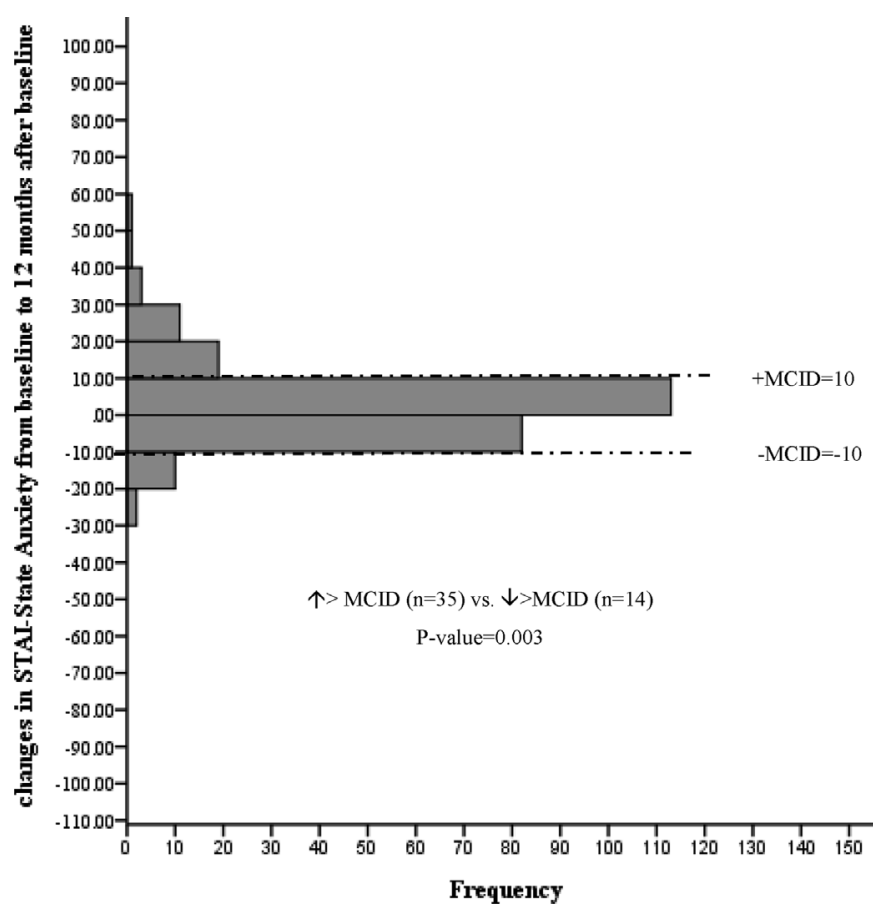

Figure 8 Changes in Spielberger STAI from baseline to 12 months among participants with a positive scan results. MCID, minimal clinically important difference; STAI, State Trait Anxiety Inventory.

not shown), an alternative distribution-based approach to MCID determination. ${ }^{40}$

The findings of our study corroborate and expand the current evidence-based information on lung cancer screening decision-making by showing that there is a minimal overall psychological impact associated with lung cancer screening. However, certain populations (ie, females, participants with higher baseline concern about lung cancer) may be at a higher risk of negative psychological impact. This suggests that an improved communication is needed throughout the entire lung cancer screening process, especially for the potentially vulnerable subgroups. Since most positive screens do not result in a lung cancer diagnosis, approaches to better define screening exam findings and reduce false positive rates could be effective in reducing the anxiety burden in this subgroup. Despite the high rate of false-positive CT results in lung cancer screening, there is no clear recommendation yet on psychological interventions to help individuals cope with abnormal CT screening results. However, literature on mammography screening has shown that immediate follow-up and consultation can significantly reduce anxieties after receiving abnormal mammograms. ${ }^{41}$

In conclusion, our study demonstrated that CT screening for lung cancer has no major impact on HRQoL among participants overall, but some individuals experience clinically significant increase in anxiety with a $\mathrm{NNH}$ of 18 at 1 year post initial screen. While these impacts may appear minor in view of the robust mortality reduction associated with LDCT screening, ongoing work to further define and minimise these negative aspects of screening is warranted given recommendations for broad screening of at-risk populations.

\section{Author affiliations}

${ }^{1}$ Department of Medicine, University of Calgary, Calgary, Alberta, Canada

${ }^{2}$ Department of Integrative Oncology, The British Columbia Cancer Agency, Vancouver, British Columbia, Canada

${ }^{3}$ Department of Respiratory Medicine, Fionna Stanley Hospital and University of Western Australia, Perth, Australia

${ }^{4}$ Department of Surgery, Beatrice Hunter Cancer Research Institute and Dalhousie University, Halifax, Canada

${ }^{5}$ Department of Oncology, The Juravinski Cancer Centre and McMaster University, Hamilton, Ontario, Canada

${ }^{6}$ Department of Medicine, The Ottawa Hospital Cancer Center, Ottawa, Ontario, Canada

${ }^{7}$ Department de Pneumologie, Institut Universitaire de Cardiologie et de Pneumologie de Québec, Québec, Canada

${ }^{8}$ Department of Medicine, Memorial University, St John's, Newfoundland and Labrador, Canada

${ }^{9}$ Princess Margaret Cancer Centre, University Health Network, Toronto, Ontario, Canada

${ }^{10}$ Department of Health Sciences, Brock University, St. Catharines, Ontario, Canada

Contributors NT led the data analysis and data interpretation. NT and AT prepared the draft manuscript. MCT and SC contributed to data analysis. AT, SCL, MCT, M-ST, SC, SA-K, AMM PM, SP, MRJ, JG, GG, GN, SM, FL, RB, GL, HS contributed to study conception and design, data acquisition and interpretation. All authors critically reviewed and approved final manuscript and agrees to be accountable for all aspects of the work

Funding The study was funded by the Terry Fox Research Institute and the Canadian Partnership Against Cancer. Additional research support provided by the Arnie Charbonneau Cancer Institute and the Alberta Cancer Foundation. Primary results of the study have been published previously.

Competing interests None declared.

Patient consent for publication Not required.

Ethics approval Multisite approval.

Provenance and peer review Not commissioned; externally peer reviewed.

Data sharing statement № additional unpublished data from the study is made available.

Open access This is an open access article distributed in accordance with the Creative Commons Attribution Non Commercial (CC BY-NC 4.0) license, which permits others to distribute, remix, adapt, build upon this work non-commercially, and license their derivative works on different terms, provided the original work is properly cited, appropriate credit is given, any changes made indicated, and the use is non-commercial. See: http://creativecommons.org/licenses/by-nc/4.0/.

\section{REFERENCES}

1. Brambilla E, Travis WD, Brennan P, et al. Lung cancer. In: Stewart BW, Wild CP, eds. World cancer report 2014: International Agency for Research on Cancer, 2014:350-61.

2. Aberle DR, Berg CD, Black WC, et al. The National Lung Screening Trial: overview and study design. Radiology 2011;258:243-53.

3. Lewin G, Morissette K, Dickinson J, et al. Recommendations on screening for lung cancer. CMAJ 2016;188:425-32.

4. Moyer VA. Screening for lung cancer: U.S. Preventive Services Task Force recommendation statement. Ann Intern Med 2014;160:330338-8.

5. Bach PB, Mirkin JN, Oliver TK, et al. Benefits and harms of CT screening for lung cancer: a systematic review. JAMA 2012;307:2418-29.

6. Patz EF, Pinsky P, Gatsonis C, et al. NLST Overdiagnosis Manuscript Writing Team. Overdiagnosis in low-dose computed tomography screening for lung cancer. JAMA Intern Med 2014;174:269-74.

7. Marshall HM, Bowman RV, Yang IA, et al. Screening for lung cancer with low-dose computed tomography: a review of current status. $J$ Thorac Dis 2013;5(Suppl 5):S524-39.

8. Wu GX, Raz DJ, Brown L, et al. Psychological burden associated with lung cancer screening: a systematic review. Clin Lung Cancer 2016;17:30053-5. 
9. Miller AB, Madalinska JB, Church T, et al. Health-related quality of life and cost-effectiveness studies in the European randomised study of screening for prostate cancer and the US Prostate, Lung, Colon and Ovary trial. Eur J Cancer 2001;37:2154-60.

10. McWilliams A, Tammemagi MC, Mayo JR, et al. Probability of cancer in pulmonary nodules detected on first screening CT. N Engl J Med 2013;369:910-9.

11. Tammemagi MC, Schmidt H, Martel S, et al. Participant selection for lung cancer screening by risk modelling (the Pan-Canadian Early Detection of Lung Cancer [PanCan] study): a single-arm, prospective study. Lancet Oncol 2017;18:1523-31.

12. Tammemagi MC, Freedman MT, Pinsky PF, et al. Prediction of true positive lung cancers in individuals with abnormal suspicious chest radiographs: a prostate, lung, colorectal, and ovarian cancer screening trial study. J Thorac Oncol 2009;4:710-21.

13. Tremblay A, Taghizadeh N, McWilliams AM, et al. Pan-Canadian Early Lung Cancer Study Group. Low prevalence of high-grade lesions detected with autofluorescence bronchoscopy in the setting of lung cancer screening in the pan-canadian lung cancer screening study. Chest 2016;150:1015-22.

14. Jenkinson $C$, Layte $R$, Jenkinson $D$, et al. A shorter form health survey: can the SF-12 replicate results from the SF-36 in longitudina studies? J Public Health Med 1997;19:179-86.

15. Bansback N, Tsuchiya A, Brazier J, et al. Canadian valuation of EQ$5 \mathrm{D}$ health states: preliminary value set and considerations for future valuation studies. PLoS One 2012;7:e31115.

16. Spielberger CD, Gorsuch RL, Lushene RE. Test manual for the statetrait anxiety inventory. Palo Alto, CA: Consulting Psychologists Press, 1970.

17. Zanini A, Aiello M, Adamo D, et al. Estimation of minimal clinically important difference in EQ-5D visual analog scale score after pulmonary rehabilitation in subjects with COPD. Respir Care 2015;60:88-95.

18. Le QA, Doctor JN, Zoellner LA, et al. Minimal clinically important differences for the EQ-5D and QWB-SA in Post-traumatic Stress Disorder (PTSD): results from a Doubly Randomized Preference Trial (DRPT). Health Qual Life Outcomes 2013:11:59.

19. Parker SL, Godil SS, Shau DN, et al. Assessment of the minimum clinically important difference in pain, disability, and quality of life after anterior cervical discectomy and fusion: clinical article. $J$ Neurosurg Spine 2013;18:154-60.

20. Corsaletti BF, Proença MGL, Bisca GKW, et al. Minimal important difference for anxiety and depression surveys after intervention to increase daily physical activity in smokers. Fisioter Pesq 2014;21:359-64.

21. Gareen IF, Duan F, Greco EM, et al. Impact of lung cancer screening results on participant health-related quality of life and state anxiety in the National Lung Screening Trial. Cancer 2014;120:3401-9.

22. van den Bergh KA, Essink-Bot ML, Borsboom GJ, et al. Longterm effects of lung cancer computed tomography screening on health-related quality of life: the NELSON trial. Eur Respir $J$ 2011;38:154-61

23. Taylor KL, Shelby R, Gelmann E, et al. Quality of life and trial adherence among participants in the prostate, lung, colorectal, and ovarian cancer screening trial. J Natl Cancer Inst 2004;96:1083-94.
24. Brain K, Lifford KJ, Carter B, et al. Long-term psychosocial outcomes of low-dose CT screening: results of the UK Lung Cancer Screening randomised controlled trial. Thorax 2016;71:996-1005.

25. Slatore CG, Sullivan DR, Pappas M, et al. Patient-centered outcomes among lung cancer screening recipients with computed tomography: a systematic review. J Thorac Oncol 2014:9:927-34.

26. Aggestrup LM, Hestbech MS, Siersma V, et al. Psychosocial consequences of allocation to lung cancer screening: a randomised controlled trial. BMJ Open 2012;2:e000663.

27. Rasmussen JF, Siersma V, Pedersen JH, et al. Psychosocial consequences in the Danish randomised controlled lung cancer screening trial (DLCST). Lung Cancer 2015;87:65-72.

28. van den Bergh KA, Essink-Bot ML, Borsboom GJ, et al. Short-term health-related quality of life consequences in a lung cancer CT screening trial (NELSON). Br J Cancer 2010;102:27-34.

29. Byrne MM, Weissfeld J, Roberts MS. Anxiety, fear of cancer, and perceived risk of cancer following lung cancer screening. Med Decis Making 2008;28:917-25.

30. Jaeschke R, Singer J, Guyatt GH. Measurement of health status. Ascertaining the minimal clinically important difference. Control Clin Trials 1989;10:407-15.

31. Tammemägi MC, Katki HA, Hocking WG, et al. Selection criteria for lung-cancer screening. N Engl J Med 2013;368:728-36.

32. Johnson JA, Pickard AS. Comparison of the EQ-5D and SF-12 health surveys in a general population survey in Alberta, Canada. Med Care 2000;38:115-21.

33. Mols F, Pelle AJ, Kupper N. Normative data of the SF-12 health survey with validation using postmyocardial infarction patients in the Dutch population. Qual Life Res 2009;18:403-14

34. Hestbech MS, Siersma V, Dirksen A, et al. Participation bias in a randomised trial of screening for lung cancer. Lung Cancer 2011;73:325-31.

35. Tramontano AC, Schrag DL, Malin JK, et al. Catalog and comparison of societal preferences (utilities) for lung cancer health states: results from the Cancer Care Outcomes Research and Surveillance (CanCORS) study. Med Decis Making 2015;35:371-87.

36. Pickard AS, De Leon MC, Kohlmann T, et al. Psychometric comparison of the standard EQ-5D to a 5 level version in cancer patients. Med Care 2007;45:259-63.

37. Wang P, Luo N, Tai ES, et al. The EQ-5D-5L is more discriminative than the EQ-5D-3L in patients with diabetes in Singapore. Value Health Reg Issues 2016;9:57-62.

38. Lo S, Andrews S. To transform or not to transform: using generalized linear mixed models to analyse reaction time data. Front Psychol 2015;6:1171.

39. Bonnetain F, Fiteni F, Efficace F, et al. Statistical challenges in the analysis of health-related quality of life in cancer clinical trials. $J$ Clin Oncol 2016;34:1953-6.

40. Norman GR, Sloan JA, Wyrwich KW. Interpretation of changes in health-related quality of life: the remarkable universality of half a standard deviation. Med Care 2003;41:582-92.

41. Barton MB, Morley DS, Moore S, et al. Decreasing women's anxieties after abnormal mammograms: a controlled trial. J Natl Cancer Inst 2004;96:529-38. 